\title{
Prostate-specific antigen test use reported in the 2000 National Health Interview Survey
}

\author{
Louie E. Ross, Ph.D., ${ }^{a, *}$ Ralph J. Coates, Ph.D., ${ }^{a}$ Nancy Breen, Ph.D., ${ }^{b}$

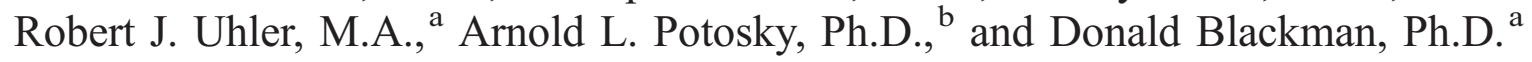 \\ ${ }^{a}$ Division of Cancer Prevention and Control, National Center for Chronic Disease Prevention and Health Promotion, \\ Centers for Disease Control and Prevention, Atlanta, GA 30341-3717, USA \\ ${ }^{\mathrm{b}}$ Division of Cancer Control and Population Sciences, National Cancer Institute, Bethesda, MD 20892, USA
}

Available online 26 February 2004

\begin{abstract}
Background. In 2000, the National Health Interview Survey (NHIS) collected information about prostate-specific antigen (PSA) test use in a representative sample of U.S. men.

Methods. This study examined PSA test use in subgroups defined by personal and social characteristics.

Results. Among men aged 50 and older with no history of prostate cancer, 56.8\% reported ever having had a PSA test, 34.1\% reported having had a screening PSA test during the previous year, and $30.0 \%$ reported having had three or more tests during the previous 5 years. Screening was greater among men aged 60-79 years, those with greater access to care, and those practicing other preventive behaviors. Among men in their 40s, use tended to be higher among African-American men.

Conclusions. The prevalence and patterns of PSA screening suggest that PSA is used like other cancer screening tests among about a third of U.S. men. Because of the lack of scientific consensus on whether prostate cancer screening is beneficial, more information is needed on how knowledgeable both patients and practitioners are about the potential benefits and harms of screening and how prostate cancer screening decisions are made.

(C) 2004 The Institute For Cancer Prevention and Elsevier Inc. All rights reserved.
\end{abstract}

Keywords: Prostate cancer; Screening; Prostate-specific antigen

\section{Introduction}

Although there is no scientific consensus that screening with the prostate-specific antigen (PSA) test reduces deaths from prostate cancer, and the debate is ongoing as to whether the potential benefits of screening outweigh the possible harms [1-6], the PSA test is commonly used [712]. Medicare claims data $[9,13]$, and surveys of clinicians [14-16], and men [7,9-11,13,17-19] in certain U.S. geographic areas or health care systems indicate that PSA testing increased during the 1980s and 1990s.

Although use has increased, there is limited published information about the prevalence of PSA test use in subpopulations of men defined by demographic or social characteristics that may influence use of PSA tests; additionally, studies do not routinely distinguish between PSA tests done

\footnotetext{
* Corresponding author. Fax: +1-770-488-3040

E-mail address: lor3@cdc.gov (L.E. Ross).
}

for screening and other purposes. Many studies are specific to a particular region or clinic, have small samples, or are not population-based [9-14,17-25]. Factors related to use of PSA testing and/or use of digital rectal examination (DRE) include age $[11,20,21,26]$, race/ethnicity $[13,14,17-$ $19,22,26]$, income/poverty status $[17,19,21,23,26]$, immigrant status [26], education $[12,20,26]$, health insurance status [12,26,27], having a regular source of healthcare $[12,21,26]$, marital status [23], prior prostate screening history [23], family history of prostate cancer [24], history of urinary tract symptoms [23,25], other illnesses [7,21], and health behaviors such as exercise and diet [11].

The 2000 National Health Interview Survey (NHIS) provides information about PSA test use among a nationally representative sample of U.S. men [26]. Our study uses these data to provide the first detailed examination of PSA use in a nationally representative sample of U.S. men. Its main purposes were to (1) examine the prevalence of annual PSA screening and routine test use; (2) describe use among 
subgroups defined by three sets of characteristics: factors found to be related to PSA test use in earlier research, factors reported to influence use of cancer screening tests in women, and some specific health-related factors that might influence decisions about PSA test use; and (3) examine use adjusted for other factors that may affect use.

\section{Study methods}

The NHIS is an annual health survey conducted by the National Center for Health Statistics [28]. The survey includes core questions about the respondent's health, demographic and socioeconomic circumstances, and access to and use of health services. It also contains one or more annual supplements addressing particular health issues. In 2000, the survey collected information related to cancer prevention and control [26]. One sample adult was randomly selected from each family participating in the NHIS. In-person interviews were conducted by trained U.S. Bureau of the Census interviewers. African-Americans and Hispanic households were over-sampled to obtain for more precise estimates [28].

\section{Study population}

Interviews were conducted with 32,374 adults in 39,264 families. In 7,784 families, there were no adult males in residence. The adult sample response rate was $72.1 \%$. Our analysis focused on men aged 50 and older because most organizations that support prostate cancer screening recommend that annual testing begin at age 50 [29]. Because African-American men and men with a family history of prostate cancer are at higher risk at younger ages, some organizations recommend offering PSA testing at an earlier age for those men [5]. Therefore, we conducted additional analyses for men in their 40s. From the sample of 5,220 men aged 50 and older, we excluded 222 men because they had prostate cancer and were likely to have PSA testing as part of their routine care and 368 men because they did not answer the PSA test use questions. For multivariate analysis, we excluded additional 287 men for whom we lacked information on other relevant variables.

\section{Data collection}

Male respondents aged 40 and older were asked if they had heard of the PSA test, and if so, whether they had one, and if they had, when their most recent test was and the reason for it. They were also asked how many tests they had received in the last 5 years. From those questions, we created four PSA test use measures indicating (1) if a man had ever had a PSA test, (2) if he had had a test in the previous year, (3) if he had had a recent PSA test for screening purposes (as a "routine test" or "screening test" or because of a family history), and (4) if he was a routine user, defined as having had three or more tests in the previous 5 years. PSA tests for any purpose were included in this variable because the NHIS did not ask respondents the reasons for having a PSA test other than the most recent one.

A set of factors that may influence test use was selected from the literature [7-30] and categorized as indicated in Table 1 . We included several other factors associated with cancer screening in women [26,30,31] that have not been examined for their relation to PSA use in men. These include occupation [32], personal history of other cancers [30], use of other health services [31], and other healthrelated behaviors [31], such as alcohol consumption and smoking. More detailed information on these variables is published elsewhere [26,28].

From 16 possible race categories used in the interview, we recorded race into five commonly used categories: white, black/African-American, American Indian/Alaska Native, Asian, and other/multiple race. Poverty levels were based on definitions of federal poverty levels developed by the Social Security Administration [33]. For 2000, the published Census Bureau poverty level for a family of four was set at $\$ 17,603$.

Access to health care was indicated by several factors including type of health insurance and usual source of care. Private insurance was defined as a private insurance plan, a single hospital service plan, a military plan, CHAMPUS, Tricare, Medicare with private supplementation, or a health maintenance organization plan. Public health insurance included Medicaid, Medicare without private supplementation, Children's Health Insurance Program, Indian Health Service, or other public assistance such as state-sponsored programs [28]. Usual source of care was determined by asking "Is there a place that you usually go when you are sick or need advice about your health?" Persons who stated that the emergency room was their usual source of care were defined as having no usual source of care.

Health status characteristics that we anticipated might influence PSA test use included family history of prostate cancer, self-assessment of general health, and an indicator of the number of chronic diseases the respondent had at the time of the interview. We computed an index of chronic diseases by summing the following self-reported conditions: hypertension, stroke, emphysema, diabetes, chronic bronchitis, failing kidneys, liver condition, and heart disease. Many organizations suggest that men with a limited life expectancy, usually less than 10 years, would not benefit from screening [29]. The presence of one or more chronic diseases and age category of 80 and older were used as indicators of possibly reduced life expectancy.

\section{Data analysis}

The NHIS used a stratified, multistage-cluster sample [28]. The statistical program SUDAAN was used in all of 
Table 1

Characteristics of men ${ }^{\mathrm{a}}$ aged 50 or older in the 2000 NHIS

\begin{tabular}{l}
\hline \\
\hline Demographics \\
Age \\
$50-64$ years \\
$65-79$ years \\
$80+$ years \\
Race \\
White \\
African-American \\
American Indian, Alaskan Native \\
Asian \\
Other \\
Ethnicity \\
Hispanic \\
Non-Hispanic \\
Region \\
Northeast \\
Midwest \\
South \\
West \\
Length of U.S. residence \\
$<10$ years \\
$\geq 10$ years \\
Born in US
\end{tabular}

\section{Socioeconomic status}

Education

$<$ High school graduate

High school graduate

Some college/tech school

College grad

Poverty threshold

$<200 \%$

$200-299 \%$

$300-399 \%$

$400-599 \%$

$500 \%+$

Unknown

Marital status

Married/living with partner

Widowed

Divorced or separated

Never married

Family size

1 person

2 persons

3 persons

4+ persons

Occupation

Executive/professional/managerial

Technicians/sales/admin support/student

Blue collar/service/homemaker

Retired

Unable to work/laid off/looking

Access to health care
Health insurance
None
Public
Private/military
Usual source of medical care
Yes
No

Table 1 (continued)

\begin{tabular}{|c|c|c|}
\hline & $N$ & $\%^{\mathrm{b}}$ \\
\hline \multicolumn{3}{|l|}{ Access to health care } \\
\hline Language spoken, among Hispanics & 552 & \\
\hline Spanish & & 48.8 \\
\hline English & & 51.2 \\
\hline $\begin{array}{l}\text { Seen MD or health care } \\
\text { provider in past year }\end{array}$ & 4921 & \\
\hline Yes & & 84.5 \\
\hline No & & 15.5 \\
\hline \multicolumn{3}{|l|}{ Health status } \\
\hline Reported health status & 4986 & \\
\hline Excellent & & 20.6 \\
\hline Very good & & 30.3 \\
\hline Good & & 29.9 \\
\hline Fair or poor & & 19.2 \\
\hline Family history of prostate cancer & 4712 & \\
\hline Yes & & 6.3 \\
\hline No & & 93.7 \\
\hline Number of chronic diseases & 4998 & \\
\hline None & & 43.5 \\
\hline 1 disease & & 31.6 \\
\hline 2 diseases & & 16.1 \\
\hline 3 diseases & & 5.8 \\
\hline $4+$ diseases & & 3.1 \\
\hline Cancer $^{\mathrm{c}}$ & 4998 & \\
\hline Yes & & 4.9 \\
\hline No & & 95.1 \\
\hline \multicolumn{3}{|l|}{ Screening } \\
\hline Had colorectal cancer screening ${ }^{\mathrm{d}}$ & 4627 & \\
\hline Yes & & 30.7 \\
\hline No & & 69.3 \\
\hline \multicolumn{3}{|l|}{ Health-related behaviors } \\
\hline Cigarette use & 4953 & \\
\hline Current (smoke everyday or some days) & & 19.5 \\
\hline Former & & 45.6 \\
\hline Never & & 34.9 \\
\hline Alcohol use & 4897 & \\
\hline Lifetime abstainer & & 17.4 \\
\hline Former drinker & & 22.5 \\
\hline Moderate drinker & & 50.6 \\
\hline $\begin{array}{l}\text { Excessive drinker } \\
\text { ( } 2 \text { or more drinks per day or } \\
5 \text { or more drinks on } 12 \text { or } \\
\text { more occasions per year) }\end{array}$ & & 9.5 \\
\hline
\end{tabular}

${ }^{a}$ Excluding men with prostate cancer.

${ }^{\mathrm{b}}$ Adjusted for the NHIS sampling methods and weighted to the U.S. census as described in Study methods.

${ }^{\mathrm{c}}$ Excluding non-melanoma skin cancer.

${ }^{\mathrm{d}}$ Fecal occult blood test in past 5 years or sigmiodoscopy, proctoscopy, or colonoscopy in last 5 years.

our analyses to account for this sampling [34]. The SUDAAN analyses used sample weights provided with the NHIS public use data file to adjust for the complex, cluster sampling, and over-sampling. Further, the analyses were weighted ("post-stratified") to the U.S. census distributions by age and race/ethnicity for the year 2000 to provide estimates representative of U.S. men in the general population [28]. We first computed the percentages of men who reported a PSA test in groups defined by the socio- 
Table 2

Percentage of men aged 50 or older who have had PSA test(s) by population subgroups

\begin{tabular}{|c|c|c|c|c|c|c|c|c|c|c|c|c|}
\hline & \multicolumn{4}{|c|}{ Had a screening PSA in the past year } & \multicolumn{4}{|c|}{ Had any PSA in the past year } & \multicolumn{4}{|c|}{ Had $3+$ PSAs in the past 5 years } \\
\hline & $N$ & $\%^{\mathrm{b}}$ & $\mathrm{PV}^{\mathrm{c}}$ & $\mathrm{CI}^{\mathrm{d}}$ & $N$ & $\%^{\mathrm{b}}$ & $\mathrm{PV}^{\mathrm{c}}$ & $\mathrm{CI}^{\mathrm{d}}$ & $N$ & $\%^{\mathrm{b}}$ & $\mathrm{PV}^{\mathrm{c}}$ & $\mathrm{CI}^{\mathrm{d}}$ \\
\hline \multicolumn{13}{|l|}{ Demographics } \\
\hline All men & 4630 & 34.1 & & $32.5-35.7$ & 4630 & 39.0 & & $37.3-40.7$ & 4569 & 29.7 & & $27.9-31.4$ \\
\hline Age & & & 0.000 & & & & 0.000 & & & & 0.000 & \\
\hline $50-64$ years & 2676 & 29.9 & & $27.7-32.1$ & 2676 & 33.2 & & $30.9-35.4$ & 2659 & 23.8 & & $21.7-26.0$ \\
\hline $65-79$ years & 1577 & 43.8 & 0.000 & $41.2-46.4$ & 1577 & 51.3 & 0.000 & $48.7-53.9$ & 1539 & 41.7 & 0.000 & $38.8-44.5$ \\
\hline $80+$ years & 377 & 26.3 & 0.189 & $21.5-31.1$ & 377 & 33.8 & 0.828 & $28.6-39.1$ & 371 & 26.5 & 0.334 & $21.5-31.6$ \\
\hline Race & & & 0.059 & & & & 0.003 & & & & 0.003 & \\
\hline White & 3859 & 34.8 & & $33.1-36.5$ & 3859 & 40.2 & & $38.5-41.9$ & 3811 & 30.9 & & $29.2-32.6$ \\
\hline African-American & 513 & 31.4 & 0.154 & $27.0-35.8$ & 513 & 34.4 & 0.017 & $29.9-38.9$ & 503 & 26.5 & 0.088 & $21.5-31.4$ \\
\hline $\begin{array}{l}\text { American Indian, } \\
\text { Alaskan Native }\end{array}$ & 32 & 18.7 & 0.002 & $8.7-28.8$ & 32 & 27.9 & 0.096 & $13.5-42.4$ & 32 & 20.3 & 0.095 & $8.0-32.7$ \\
\hline Asian & 104 & 21.8 & 0.001 & $14.0-29.6$ & 104 & 24.9 & 0.000 & $18.1-31.8$ & 103 & 18.5 & 0.000 & $12.0-24.9$ \\
\hline Other & 122 & 28.8 & 0.172 & $20.3-37.3$ & 122 & 32.0 & 0.085 & $22.8-41.2$ & 120 & 16.8 & 0.001 & $8.9-24.7$ \\
\hline Ethnicity & & & 0.000 & & & & 0.000 & & & & 0.000 & \\
\hline Hispanic & 538 & 24.4 & 0.000 & $19.8-29.1$ & 538 & 28.9 & 0.000 & $24.1-33.8$ & 535 & 17.9 & 0.000 & $14.0-21.9$ \\
\hline Non-Hispanic & 4092 & 34.9 & & $33.2-36.5$ & 4092 & 40.0 & & $38.3-41.7$ & 4034 & 30.9 & & $29.2-32.6$ \\
\hline Region & & & 0.072 & & & & 0.025 & & & & 0.040 & \\
\hline Northeast & 869 & 34.4 & & $30.9-38.0$ & 869 & 41.0 & & $37.4-44.6$ & 850 & 31.7 & & $27.5-36.0$ \\
\hline Midwest & 1074 & 35.2 & 0.721 & $32.7-37.8$ & 1074 & 40.0 & 0.657 & $37.4-42.6$ & 1059 & 28.6 & 0.235 & $25.9-31.4$ \\
\hline South & 1701 & 35.5 & 0.650 & $32.7-38.2$ & 1701 & 40.3 & 0.765 & $37.3-43.2$ & 1681 & 32.0 & 0.902 & $29.3-34.8$ \\
\hline West & 986 & 30.1 & 0.111 & $26.2-34.1$ & 986 & 34.9 & 0.022 & $31.2-38.6$ & 979 & 26.6 & 0.062 & $23.3-29.9$ \\
\hline Length of U.S. residence & & & 0.000 & & & & 0.000 & & & & 0.000 & \\
\hline$<10$ years & 62 & 12.3 & 0.000 & $2.2-22.4$ & 62 & 17.4 & 0.000 & $5.9-28.9$ & 62 & 13.5 & 0.001 & $3.6-23.4$ \\
\hline$\geq 10$ years & 492 & 24.5 & 0.000 & $19.9-29.1$ & 492 & 30.3 & 0.000 & $25.2-35.3$ & 489 & 22.0 & 0.001 & $17.1-27.0$ \\
\hline Born in US & 4028 & 35.5 & & $33.8-37.1$ & 4028 & 40.6 & & $38.9-42.3$ & 3971 & 31.2 & & $29.5-33.0$ \\
\hline \multicolumn{13}{|l|}{ Socioeconomic status } \\
\hline Education & & & 0.000 & & & & 0.000 & & & & 0.000 & \\
\hline$<$ High school graduate & 1234 & 22.9 & & $20.3-25.5$ & 1234 & 27.3 & & $24.7-30.0$ & 1224 & 17.9 & & $15.4-20.5$ \\
\hline High school graduate & 1257 & 32.2 & 0.000 & $29.2-35.1$ & 1257 & 36.9 & 0.000 & $33.8-39.9$ & 1240 & 27.2 & 0.000 & $24.4-30.0$ \\
\hline Some college/tech school & 1017 & 39.5 & 0.000 & $36.0-42.9$ & 1017 & 44.4 & 0.000 & $41.0-47.8$ & 998 & 35.7 & 0.000 & $32.2-39.1$ \\
\hline College grad & 1078 & 43.3 & 0.000 & $39.4-47.3$ & 1078 & 50.2 & 0.000 & $46.5-53.9$ & 1066 & 41.4 & 0.000 & $38.1-44.8$ \\
\hline Poverty threshold & & & 0.000 & & & & 0.000 & & & & 0.000 & \\
\hline$<200 \%$ & 1056 & 25.7 & & $22.8-28.6$ & 1056 & 29.7 & & $26.6-32.8$ & 1,046 & 19.7 & & $16.7-22.8$ \\
\hline $200-299 \%$ & 579 & 27.9 & 0.380 & $24.1-31.7$ & 579 & 31.8 & 0.404 & $27.9-35.8$ & 574 & 24.1 & 0.080 & $20.3-28.0$ \\
\hline $300-399 \%$ & 411 & 31.1 & 0.069 & $26.5-35.7$ & 411 & 38.7 & 0.003 & $33.9-43.6$ & 407 & 30.6 & 0.000 & $25.9-35.3$ \\
\hline $400-499 \%$ & 357 & 33.5 & 0.022 & $27.4-39.6$ & 357 & 40.8 & 0.003 & $34.3-47.2$ & 353 & 31.4 & 0.000 & $26.4-36.5$ \\
\hline $500 \%+$ & 1062 & 45.6 & 0.000 & $41.4-49.9$ & 1062 & 52.1 & 0.000 & $48.0-56.1$ & 1058 & 43.0 & 0.000 & $39.3-46.8$ \\
\hline Unknown & 1165 & 33.5 & 0.000 & $30.5-36.5$ & 1165 & 38.0 & 0.000 & $34.8-41.1$ & 1131 & 29.7 & 0.000 & $26.5-32.9$ \\
\hline Marital status & & & 0.000 & & & & 0.000 & & & & 0.000 & \\
\hline Married/living with partner & 2999 & 36.0 & & $34.0-38.0$ & 2999 & 41.4 & & $39.4-43.4$ & 2965 & 31.8 & & $29.8-33.8$ \\
\hline Widowed & 501 & 31.1 & 0.148 & $24.7-37.4$ & 501 & 36.8 & 0.196 & $30.2-43.4$ & 486 & 24.0 & 0.004 & $19.2-28.7$ \\
\hline Divorced or separated & 790 & 24.6 & 0.000 & $20.5-28.8$ & 790 & 27.9 & 0.000 & $23.9-32.0$ & 781 & 20.5 & 0.000 & $17.0-24.1$ \\
\hline Never married & 331 & 30.7 & 0.099 & $24.7-36.7$ & 331 & 35.6 & 0.079 & $29.5-41.7$ & 328 & 23.3 & 0.004 & $17.9-28.7$ \\
\hline Family size & & & 0.000 & & & & 0.000 & & & & 0.000 & \\
\hline 1 person & 1497 & 28.4 & & $26.0-30.9$ & 1497 & 32.8 & & $30.2-35.3$ & 1471 & 25.6 & & $23.1-28.1$ \\
\hline 2 persons & 2281 & 36.6 & 0.000 & $34.4-38.7$ & 2281 & 42.4 & 0.000 & $40.3-44.5$ & 2253 & 32.8 & 0.000 & $30.8-34.9$ \\
\hline 3 persons & 481 & 35.0 & 0.010 & $30.6-39.5$ & 481 & 38.2 & 0.037 & $33.6-42.8$ & 475 & 24.2 & 0.568 & $20.0-28.3$ \\
\hline $4+$ persons & 371 & 28.8 & 0.915 & $22.5-35.1$ & 371 & 32.6 & 0.971 & $26.2-39.1$ & 370 & 26.4 & 0.819 & $19.9-32.9$ \\
\hline Occupation & & & 0.000 & & & & 0.000 & & & & 0.000 & \\
\hline $\begin{array}{l}\text { Executive/professional/ } \\
\text { managerial }\end{array}$ & 694 & 40.8 & & $35.1-46.6$ & 694 & 43.9 & & $38.2-49.6$ & 688 & 38.8 & & $33.1-44.5$ \\
\hline $\begin{array}{l}\text { Technicians/sales/ } \\
\text { admin support/student }\end{array}$ & 426 & 38.9 & 0.667 & $32.3-45.5$ & 426 & 45.9 & 0.599 & $41.3-50.4$ & 423 & 36.6 & 0.624 & $29.7-43.4$ \\
\hline $\begin{array}{l}\text { Blue collar/service/ } \\
\text { homemaker }\end{array}$ & 1067 & 27.9 & 0.001 & $23.0-32.7$ & 1067 & 30.8 & 0.001 & $26.0-35.7$ & 1058 & 18.7 & 0.000 & $14.9-22.4$ \\
\hline Retired & 1823 & 39.8 & & $35.0-44.5$ & 1823 & 44.5 & & $39.8-49.2$ & 1782 & 33.6 & 0.139 & $29.5-37.7$ \\
\hline $\begin{array}{l}\text { Unable to work/ } \\
\text { laid off/looking }\end{array}$ & 523 & 25.2 & 0.000 & $20.5-30.0$ & 523 & 30.4 & 0.000 & $25.3-35.5$ & 522 & 21.1 & 0.000 & $16.7-25.4$ \\
\hline
\end{tabular}


Table 2 (continued)

\begin{tabular}{|c|c|c|c|c|c|c|c|c|c|c|c|c|}
\hline & \multicolumn{4}{|c|}{ Had a screening PSA in the past year } & \multicolumn{4}{|c|}{ Had any PSA in the past year } & \multicolumn{4}{|c|}{ Had $3+$ PSAs in the past 5 years } \\
\hline & $N$ & $\%{ }^{\mathrm{b}}$ & $\mathrm{PV}^{\mathrm{c}}$ & $\mathrm{CI}^{\mathrm{d}}$ & $N$ & $\%{ }^{\mathrm{b}}$ & $\mathrm{PV}^{\mathrm{c}}$ & $\mathrm{CI}^{\mathrm{d}}$ & $N$ & $\%{ }^{b}$ & $\mathrm{PV}^{\mathrm{c}}$ & $\mathrm{CI}^{\mathrm{d}}$ \\
\hline \multicolumn{13}{|l|}{ Access to health care } \\
\hline Health insurance & & & 0.000 & & & & 0.000 & & & & 0.000 & \\
\hline None & 378 & 8.0 & 0.000 & $4.2-11.7$ & 378 & 9.7 & 0.000 & $5.7-13.6$ & 378 & 6.0 & 0.000 & $2.6-9.5$ \\
\hline Public & 861 & 26.8 & 0.000 & $22.9-30.8$ & 861 & 31.6 & 0.000 & $27.5-35.7$ & 850 & 20.7 & 0.000 & $16.9-24.5$ \\
\hline Private/military & 3374 & 37.4 & & $35.4-39.3$ & 3374 & 42.7 & & $40.7-44.6$ & 3325 & 34.0 & & $32.1-36.0$ \\
\hline $\begin{array}{l}\text { Usual source of } \\
\text { medical care }\end{array}$ & & & 0.000 & & & & 0.000 & & & & 0.000 & \\
\hline Yes & 4148 & 36.3 & & $34.6-38.0$ & 4148 & 41.6 & & $40.0-43.3$ & 4086 & 31.9 & & $30.2-33.6$ \\
\hline No & 479 & 9.5 & 0.000 & $6.1-12.9$ & 479 & 11.4 & 0.000 & $7.6-15.2$ & 480 & 8.2 & 0.000 & $4.9-11.6$ \\
\hline $\begin{array}{l}\text { Language spoken, } \\
\text { among Hispanics }\end{array}$ & & & 0.043 & & & & 0.004 & & & & 0.000 & \\
\hline Spanish & 274 & 20.0 & 0.070 & $14.3-25.8$ & 274 & 22.9 & 0.014 & $17.0-28.9$ & 273 & 9.8 & 0.000 & $6.7-13.0$ \\
\hline English & 262 & 28.5 & & $21.5-35.4$ & 262 & 34.7 & & $27.5-41.8$ & 260 & 25.4 & & $18.9-31.9$ \\
\hline $\begin{array}{l}\text { Seen an MD or HCP } \\
\text { in past } 12 \text { months }\end{array}$ & & & 0.000 & & & & 0.000 & & & & 0.000 & \\
\hline Yes & 3809 & 38.6 & & $36.8-40.3$ & 3809 & 44.2 & & $42.4-46.0$ & 3750 & 33.7 & & $31.8-35.6$ \\
\hline No & 792 & 9.1 & 0.000 & $6.5-11.8$ & 792 & 10.8 & 0.000 & $7.8-13.7$ & 791 & 9.8 & 0.000 & $6.9-12.6$ \\
\hline \multicolumn{13}{|l|}{ Health status } \\
\hline Reported health status & & & 0.012 & & & & 0.180 & & & & 0.001 & \\
\hline Excellent & 915 & 37.0 & & $33.4-40.6$ & 915 & 41.4 & & $37.7-45.1$ & 903 & 33.8 & & $30.3-37.4$ \\
\hline Very good & 1345 & 36.8 & 0.914 & $33.6-39.9$ & 1345 & 41.3 & 0.950 & $38.2-44.4$ & 1331 & 33.7 & 0.964 & $30.7-36.7$ \\
\hline Good & 1396 & 32.7 & 0.060 & $29.9-35.5$ & 1396 & 37.9 & 0.133 & $35.1-40.7$ & 1373 & 27.0 & 0.003 & $24.2-29.8$ \\
\hline Fair or poor & 964 & 29.6 & 0.003 & $26.4-32.9$ & 964 & 36.6 & 0.070 & $33.2-40.0$ & 952 & 26.4 & 0.003 & $23.1-29.7$ \\
\hline $\begin{array}{l}\text { Family history of } \\
\text { prostate cancer }\end{array}$ & & & 0.001 & & & & 0.000 & & & & 0.001 & \\
\hline Yes & 263 & 44.4 & 0.002 & $37.5-51.2$ & 263 & 53.7 & 0.000 & $46.7-60.7$ & 258 & 40.3 & 0.002 & $33.7-47.0$ \\
\hline No & 4,294 & 33.4 & & $31.8-35.1$ & 4294 & 38.3 & & $36.6-40.0$ & 4245 & 29.5 & & $27.8-31.2$ \\
\hline Number of chronic diseases & & & 0.000 & & & & 0.000 & & & & 0.000 & \\
\hline None & 1966 & 29.1 & & $26.7-31.5$ & 1966 & 34.2 & & $31.7-36.7$ & 1946 & 26.6 & & $24.2-29.0$ \\
\hline 1 disease & 1470 & 37.3 & 0.000 & $34.5-40.1$ & 1470 & 41.3 & 0.000 & $38.6-44.1$ & 1450 & 30.6 & 0.019 & $28.0-33.1$ \\
\hline 2 diseases & 774 & 40.1 & 0.000 & $36.3-44.0$ & 774 & 45.9 & 0.000 & $41.7-50.0$ & 761 & 37.5 & 0.000 & $33.4-41.6$ \\
\hline 3 diseases & 271 & 34.2 & 0.182 & $27.2-41.2$ & 271 & 42.3 & 0.046 & $34.7-49.9$ & 270 & 29.4 & 0.444 & $22.6-36.2$ \\
\hline $4+$ diseases & 149 & 40.8 & 0.032 & $30.4-51.3$ & 149 & 48.5 & 0.011 & $37.8-59.1$ & 142 & 28.3 & 0.701 & $19.9-36.7$ \\
\hline Cancer $^{\mathrm{e}}$ & & & 0.389 & & & & 0.344 & & & & 0.135 & \\
\hline Yes & 227 & 31.6 & 0.513 & $23.9-39.4$ & 227 & 45.1 & 0.180 & $36.7-53.5$ & 222 & 33.2 & 0.347 & $26.4-40.0$ \\
\hline No & 4403 & 34.3 & & $32.7-35.9$ & 4403 & 39.1 & & $37.5-40.8$ & 4347 & 29.8 & & $28.2-31.5$ \\
\hline \multicolumn{13}{|l|}{ Screening } \\
\hline Had colorectal screening $^{\mathrm{f}}$ & & & 0.000 & & & & 0.000 & & & & 0.000 & \\
\hline Yes & 1308 & 57.3 & 0.000 & $54.2-60.4$ & 1308 & 61.9 & 0.000 & $58.8-64.9$ & 1289 & 47.4 & 0.000 & $44.2-50.7$ \\
\hline No & 3209 & 24.3 & & $22.6-26.0$ & 3209 & 29.7 & & $27.9-31.5$ & 3178 & 22.4 & & $20.7-24.0$ \\
\hline \multicolumn{13}{|l|}{ Health-related behaviors } \\
\hline Cigarette use & & & 0.000 & & & & 0.000 & & & & 0.000 & \\
\hline $\begin{array}{l}\text { Current (smoke everyday } \\
\text { or some days) }\end{array}$ & 962 & 27.1 & 0.001 & $23.4-30.8$ & 962 & 30.2 & 0.000 & $26.3-34.1$ & 954 & 19.3 & 0.000 & $15.9-22.8$ \\
\hline Former & 2088 & 36.3 & 0.551 & $34.1-38.6$ & 2088 & 41.6 & .547 & $39.3-44.0$ & 2062 & 32.1 & 0.970 & $29.9-34.3$ \\
\hline Never & 1569 & 35.3 & & $32.5-38.0$ & 1569 & 40.5 & & $37.7-43.2$ & 1543 & 32.2 & & $29.5-34.9$ \\
\hline Alcohol Use & & & 0.001 & & & & 0.000 & & & & 0.000 & \\
\hline Lifetime abstainer & 791 & 29.5 & & $26.0-32.9$ & 791 & 33.0 & & $29.5-36.6$ & 775 & 23.5 & & $20.3-26.8$ \\
\hline Former drinker & 1083 & 31.2 & 0.914 & $27.9-34.5$ & 1083 & 35.7 & 0.950 & $32.3-39.1$ & 1064 & 26.7 & 0.964 & $23.6-29.8$ \\
\hline Moderate drinker & 2251 & 37.7 & 0.060 & $35.3-40.2$ & 2251 & 44.3 & 0.133 & $41.9-46.7$ & 2229 & 35.3 & 0.003 & $33.0-37.5$ \\
\hline Excessive drinker ${ }^{g}$ & 468 & 33.4 & 0.003 & $28.4-38.4$ & 468 & 35.1 & 0.070 & $30.1-40.1$ & 466 & 26.3 & 0.003 & $21.2-31.4$ \\
\hline \multicolumn{13}{|c|}{${ }^{\mathrm{a}}$ Excluding men with prostate cancer. } \\
\hline \multirow{2}{*}{\multicolumn{13}{|c|}{$\begin{array}{l}{ }^{\mathrm{c}} \mathrm{Adjusted} \text { for the NHIS sampling methods and weighted to the U.S. census as described in Study methods. } \\
\text { reference level. The reference levels are blank. }\end{array}$}} \\
\hline & & & & & & & & & & & & \\
\hline \multicolumn{13}{|c|}{ d $95 \%$ Confidence limits. } \\
\hline \multicolumn{13}{|c|}{${ }^{\mathrm{e}}$ Excluding non-melanoma skin cancer. } \\
\hline${ }^{\mathrm{f}}$ Fecal occult blood test in $\mathrm{p}$ & & & & & & & & & & & & \\
\hline
\end{tabular}


Table 3

Percentage $^{\mathrm{a}}$ of men $^{\mathrm{b}}$ aged 50 or older who had PSA test(s), adjusting for all population subgroups, by population subgroups

\begin{tabular}{|c|c|c|c|c|c|c|}
\hline & \multicolumn{3}{|c|}{ Had a screening PSA in the past year, $n=4343$} & \multicolumn{3}{|c|}{ Had $3+$ PSAs in the past 5 years, $n=4296$} \\
\hline & $\%$ & $\mathrm{CI}^{\mathrm{c}}$ & $P \mathrm{PV}^{\mathrm{d}}$ & $\%$ & $\mathrm{CI}^{\mathrm{c}}$ & $P V^{d}$ \\
\hline \multicolumn{7}{|l|}{ Demographics } \\
\hline Age & & & 0.000 & & & 0.000 \\
\hline $50-64$ years & 31.6 & $29.0-34.1$ & & 26.3 & $23.7-28.9$ & \\
\hline $65-79$ years & 41.0 & $37.8-44.2$ & 0.000 & 38.1 & $34.7-41.5$ & 0.000 \\
\hline $80+$ years & 27.0 & $21.4-32.5$ & 0.182 & 25.0 & $19.6-30.5$ & 0.705 \\
\hline Race & & & 0.277 & & & 0.550 \\
\hline White & 34.0 & $32.2-35.8$ & & 30.0 & $28.2-31.8$ & \\
\hline African-American & 38.4 & $32.3-44.4$ & 0.161 & 33.8 & $26.9-40.6$ & 0.275 \\
\hline Other & 37.2 & $28.9-45.6$ & 0.448 & 29.5 & $21.1-37.9$ & 0.915 \\
\hline Ethnicity & & & 0.323 & & & 0.469 \\
\hline Hispanic & 37.4 & $31.4-43.4$ & 0.324 & 32.5 & $26.3-38.6$ & 0.469 \\
\hline Non-Hispanic & 34.3 & $32.5-36.0$ & & 30.1 & $28.3-32.0$ & \\
\hline Region & & & 0.034 & & & 0.001 \\
\hline Northeast & 33.8 & $30.1-37.5$ & & 31.3 & $27.2-35.4$ & \\
\hline Midwest & 35.1 & $32.3-37.9$ & 0.597 & 28.3 & $25.4-31.2$ & 0.227 \\
\hline South & 36.9 & $33.9-39.9$ & 0.207 & 33.7 & $30.8-36.6$ & 0.357 \\
\hline West & 29.6 & $25.8-33.4$ & 0.111 & 25.2 & $21.8-28.6$ & 0.018 \\
\hline Length of U.S. residence & & & 0.016 & & & 0.059 \\
\hline$<10$ years & 18.6 & $2.2-35.0$ & 0.113 & 18.0 & $5.0-31.0$ & 0.108 \\
\hline$\geq 10$ years & 27.8 & $22.0-33.5$ & 0.022 & 24.5 & $18.4-30.6$ & 0.070 \\
\hline Born in US & 35.2 & $33.4-37.0$ & & 30.8 & $28.9-32.7$ & \\
\hline \multicolumn{7}{|l|}{ Socioeconomic status } \\
\hline Education & & & 0.002 & & & 0.000 \\
\hline$<$ High school graduate & 28.1 & $24.5-31.7$ & & 23.1 & $19.7-26.5$ & \\
\hline High school graduate & 33.8 & $30.6-36.9$ & 0.022 & 29.7 & $26.6-32.7$ & 0.004 \\
\hline Some college/tech school & 37.3 & $34.0-40.7$ & 0.001 & 32.1 & $28.9-35.4$ & 0.000 \\
\hline College grad & 37.5 & $33.7-41.2$ & 0.001 & 34.5 & $30.8-38.2$ & 0.000 \\
\hline Poverty threshold & & & 0.026 & & & 0.013 \\
\hline$<200 \%$ & 31.6 & $27.7-35.6$ & & 25.0 & $21.0-29.0$ & \\
\hline $200-299 \%$ & 30.9 & $26.8-35.0$ & 0.792 & 26.8 & $22.7-31.0$ & 0.507 \\
\hline $300-399 \%$ & 32.3 & $27.4-37.2$ & 0.845 & 31.4 & $26.5-36.4$ & 0.044 \\
\hline $400-599 \%$ & 32.6 & $26.8-38.5$ & 0.779 & 28.9 & $23.8-34.0$ & 0.234 \\
\hline $500 \%+$ & 39.6 & $36.0-43.2$ & 0.007 & 34.3 & $31.0-37.6$ & 0.001 \\
\hline Unknown & 33.7 & $30.7-36.7$ & 0.392 & 30.9 & $27.6-34.1$ & 0.019 \\
\hline Marital status & & & 0.069 & & & 0.057 \\
\hline Married/living with partner & 35.3 & $33.2-37.3$ & & 31.0 & $29.0-33.0$ & \\
\hline Widowed & 30.1 & $25.7-34.5$ & 0.042 & 26.4 & $22.4-30.3$ & 0.039 \\
\hline Divorced or separated & 30.5 & $26.5-34.5$ & 0.044 & 27.7 & $23.4-31.9$ & 0.151 \\
\hline Never married & 34.0 & $27.9-40.1$ & 0.700 & 26.2 & $21.1-31.3$ & 0.089 \\
\hline Occupation & & & 0.001 & & & 0.000 \\
\hline Executive/professional/managerial & 35.7 & $31.3-40.0$ & & 28.9 & $25.0-32.9$ & \\
\hline Technicians/sales/admin support/student & 33.2 & $28.3-38.0$ & 0.451 & 29.3 & $24.5-34.2$ & 0.896 \\
\hline Blue collar/service/homemaker & 27.3 & $24.0-30.7$ & 0.003 & 20.2 & $16.8-23.6$ & 0.001 \\
\hline Retired & 38.4 & $35.2-41.6$ & 0.352 & 36.1 & $33.0-39.2$ & 0.006 \\
\hline Unable to work/laid off/looking & 32.6 & $26.4-38.7$ & 0.421 & 30.9 & $25.0-36.7$ & 0.591 \\
\hline \multicolumn{7}{|l|}{ Access to health care } \\
\hline Health insurance & & & 0.006 & & & 0.002 \\
\hline None & 21.6 & $13.8-29.3$ & 0.003 & 20.5 & $12.9-28.2$ & 0.013 \\
\hline Public & 31.7 & $27.4-36.1$ & 0.137 & 25.1 & $21.2-29.1$ & 0.006 \\
\hline Private/military & 35.5 & $33.6-37.4$ & & 31.6 & $29.6-33.5$ & \\
\hline Usual source of care? & & & 0.000 & & & 0.000 \\
\hline Yes & 35.8 & $34.0-37.6$ & & 31.3 & $29.5-33.2$ & \\
\hline No & 13.6 & $9.0-18.2$ & 0.000 & 12.3 & $7.7-16.9$ & 0.000 \\
\hline \multicolumn{7}{|l|}{ Health status } \\
\hline Reported health status & & & 0.274 & & & 0.040 \\
\hline Excellent & 35.2 & $31.6-38.7$ & & 32.0 & $28.6-35.4$ & \\
\hline Very good & 36.8 & $33.6-39.9$ & 0.467 & 33.1 & $30.0-36.2$ & 0.600 \\
\hline Good & 32.9 & $29.9-35.9$ & 0.341 & 27.3 & $24.4-30.2$ & 0.031 \\
\hline Fair or poor & 32.1 & $28.1-36.2$ & 0.305 & 28.1 & $24.2-32.0$ & 0.158 \\
\hline
\end{tabular}


Table 3 (continued)

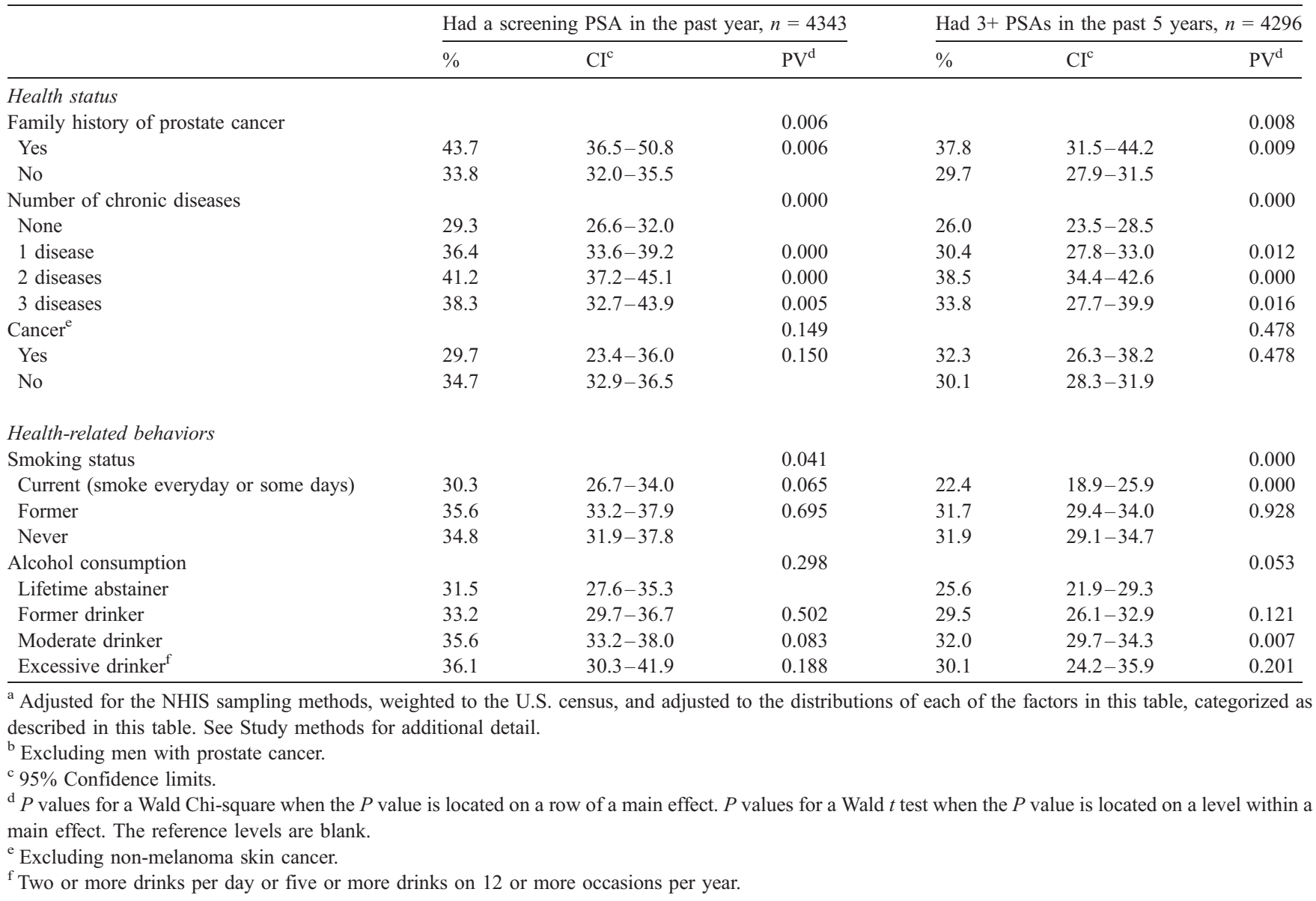

demographic, health, and health care characteristics anticipated to influence use. We used $95 \%$ confidence intervals to assess variability in the estimates of these percentages and to make general comparisons across groups. The Wald chisquare test was used first to assess the overall statistical significance of each factor, such as education or health insurance in predicting PSA test use after adjusting for age. Then, general linear contrasts were used to assess the statistical significance of differences among men within the categories of the factors, for example, comparing use in men with less than a high school level of education with use in college graduates.

We used logistic regression [35] in SUDAAN [34] to estimate the percentages of men receiving PSA testing take into account the complex sampling methods, to weight the results to the Census population characteristics by age and race/ethnicity in 2000, and to further adjust for other characteristics found to be related to PSA test use. The adjusted percentages (i.e., predicted marginals) were directly standardized to the distribution of the different categories of the other variables in the logistic models [36]. These adjusted percentages do not reflect actual use in the population, but they allow comparisons of use across categories of the variables included in the model. All models were assessed for multicollinearity. Although the final model contained a large number of factors predictive of PSA test use, we did not adjust for multiple comparisons because the factors were selected in advance based on the cancer screening literature and because use of these adjustments in observational studies increases the likelihood of excluding true associations [37].

For the multivariate logistic regression model, we excluded some variables and collapsed some categories. Asian, American Indian/Alaska Native, and other were combined into a single category because of the small numbers in each of these groups. Language spoken was dropped because it was asked only of Hispanics. Having seen a medical doctor or healthcare professional in the past 12 months was not included because of its strong association with the other health care access variables. Family size was excluded because it is a component of the poverty level and because no relation was found between family size and PSA test use in the multivariate model.

Since effects of race and ethnicity may be difficult to separate from socioeconomic circumstances, we tested for interactions among those factors, including combinations of age, race, ethnicity, level of education, poverty ratio, number of chronic illnesses, and family history. 


\section{Results}

The distribution of characteristics of the NHIS sample of men aged 50 and older (Table 1) reflected patterns in the U.S. population in 2000 [28], after adjustment for sampling methods and weighting to U.S. census distributions by age and race/ethnicity.

Use of the PSA test for screening purposes was more common among men who were aged 65-79, non-Hispanic and U.S. born (Table 2). Screening PSA test use tended to be higher among men with higher levels of socioeconomic status, that is, those with higher levels of education and income, while it was lower among men who were blue collar/service workers or unable to work, laid off, or looking for work. Screening PSA use was more common among men with greater access to healthcare, as indicated by having health insurance, a usual source of healthcare, a recent doctor visit, and use of the English language. Use was also higher in married men and those living in two or threeperson families. Use was lower among those who reported worse health, no family history, and no chronic health conditions. Men who had not been recently screened for colorectal cancer and who were current smokers had lower levels of PSA screening use, and men who reported moderate drinking were more likely to report PSA screening. Rates of PSA screening were higher than we expected among men who might not benefit from it, including men aged 80 and older $(26 \%)$, men who reported being in fair or poor health $(30 \%)$, and men with four or more chronic illnesses (41\%).

Patterns of screening PSA test use adjusted for other characteristics in the multivariable model (Table 3) were generally similar to patterns of screening use observed in the data adjusted only for sampling methods and weighted to the U.S. population (Table 2). No statistically significant interaction effects were found. However, some differences among groups were reduced and some characteristics related to use were no longer statistically significant. These included Hispanic ethnicity, reported health status, and alcohol consumption.

Relationships between routine PSA test use and the characteristics we examined were generally quite similar to relationships between screening PSA test use and those characteristics.

Among men in their 40s, screening PSA test use was somewhat higher among African-American men than among white men (Table 4). Because the numbers of men in their $40 \mathrm{~s}$ in the other racial groups were small, confidence intervals were wide or could not be calculated. Among men in their 50s, screening PSA test use was lower among Asian and American Indian/Alaska Native men than among white men. Differences in ever having had a PSA test for any purpose were similar to those observed in screening PSA use except for men in their 50s; in this group, use was lower among African-American men than among white men.

\section{Discussion}

Approximately one-third (34\%) of men aged 50 and older interviewed in the 2000 NHIS had had a screening PSA test during the last year, despite the uncertainty about the benefits of screening. By comparison, 31\% of men aged 50 and older in the 2000 NHIS had received a screening endoscopy within the previous 5 years or a fecal occult blood test (FOBT) within the previous year, the recommended intervals for colorectal cancer screening tests. Thus, PSA test use for screening is as common as screening for colorectal cancer, a procedure for which there is much stronger evidence of effectiveness and better agreement on screening [38-40]. This lack of consistency between strength of evidence and use of screening tests for prostate and colorectal cancers was borne out in another recent survey that also found prostate cancer screening to be more common among men than colorectal screening [7].

Levels of PSA test use reported in the literature in a variety of samples of men [7,9-12,17] are generally consistent with what was self-reported in the NHIS population. A study of 1998 Medicare claims data on U.S. men aged 65 and older with no prior diagnosis of prostate cancer found that approximately $38 \%$ of white men and $31 \%$ of AfricanAmerican men received annual PSA tests [9]. Telephone surveys conducted in New York [10] and Texas [12,17] in the mid-1990s found that $60 \%$ of men aged 50 and older and $37 \%$ of men aged 40 and older reported ever having had a PSA test. A similar survey in Washington State found that of men aged 40 and older, approximately 30\% reported having received a PSA test during the previous 2 years [11]. A survey of men aged 50 and older in all 50 states found that $75 \%$ had had at least one PSA test [7]. None of these studies appears to have distinguished screening PSA tests from diagnostic tests.

In this national sample, patterns of PSA test use were similar to those reported in other studies [10,11,13,14,17, 18,20-23,27]. These relationships include those we observed for income/poverty status [17,19,21,23], education [20], health insurance [12,26,27], a regular source of healthcare [12,21,26], and marital status[23]. These relations may be explained by greater access to health care due in part to greater resources and knowledge.

Although levels of PSA test use in men are lower than mammography and Pap test use in women [26], patterns of PSA test use are similar to patterns of use of the other cancer screening tests among women [26,30,31,41]. Women who report greater use of screening tests are in higher status occupations, are more likely to have access to or use other health services, and are more likely to practice healthy behaviors such as avoiding cigarette use and excessive alcohol consumption than are women who use screening tests less frequently.

We had anticipated that use of PSA as a screening test might decline among men with a greater number of chronic diseases and in fair or poor health, because screening and 
Table 4

Percentage of men ${ }^{\mathrm{a}}$ who have had PSA test(s) by age, race, and family history of prostate cancer

\begin{tabular}{|c|c|c|c|c|c|c|c|c|c|c|c|c|c|c|c|c|}
\hline & \multicolumn{4}{|c|}{$40-44$ years old } & \multicolumn{4}{|c|}{$45-49$ years old } & \multicolumn{4}{|c|}{$50+$ years old } & \multicolumn{4}{|l|}{ Total } \\
\hline & $N$ & $\%^{\mathrm{b}}$ & $\mathrm{PV}^{\mathrm{c}}$ & $\mathrm{CI}^{\mathrm{d}}$ & $N$ & $\%^{\mathrm{b}}$ & $\mathrm{PV}^{\mathrm{c}}$ & $\mathrm{CI}^{\mathrm{d}}$ & $N$ & $\%^{\mathrm{b}}$ & $\mathrm{PV}^{\mathrm{c}}$ & $\mathrm{CI}^{\mathrm{d}}$ & $N$ & $\%^{\mathrm{b}}$ & $\mathrm{PV}^{\mathrm{c}}$ & $\mathrm{CI}^{\mathrm{d}}$ \\
\hline \multicolumn{17}{|c|}{ Had any screening PSA test in the past year } \\
\hline All & 1,417 & 7.3 & & $5.8-8.8$ & 1274 & 11.3 & & $9.3-13.3$ & 4630 & 34.1 & & $32.5-35.7$ & 7321 & 25.2 & & $24.0-26.3$ \\
\hline \multicolumn{17}{|l|}{ Race } \\
\hline White & 1132 & 6.7 & & $5.1-8.3$ & 1019 & 10.4 & & $8.2-12.7$ & 3859 & 34.8 & & $33.1-36.5$ & 6010 & 25.4 & & $24.2-26.6$ \\
\hline African-American & 158 & 14.9 & 0.029 & $7.9-22.0$ & 164 & 16.8 & 0.055 & $10.8-22.8$ & 513 & 31.4 & 0.154 & $27.0-35.8$ & 835 & 25.8 & 0.803 & $22.2-29.5$ \\
\hline $\begin{array}{l}\text { American Indian, } \\
\text { Alaskan Native }\end{array}$ & 10 & 0.0 & e & e & 11 & 35.8 & 0.155 & $1.0-70.6$ & 32 & 18.7 & 0.002 & $8.7-28.8$ & 53 & 18.0 & 0.132 & $8.4-27.6$ \\
\hline Asian & 40 & 2.9 & 0.202 & $0.0-8.5$ & 32 & 11.2 & 0.875 & $1.3-21.2$ & 104 & 21.8 & 0.001 & $14.0-29.6$ & 176 & 16.2 & 0.001 & $10.9-21.6$ \\
\hline Other & 77 & 6.2 & 0.880 & $0.4-12.0$ & 48 & 7.4 & 0.446 & $0.0-14.8$ & 122 & 28.8 & 0.172 & $20.3-37.3$ & 247 & 20.6 & 0.110 & $15.0-26.3$ \\
\hline \multicolumn{17}{|l|}{$\begin{array}{l}\text { Family history of } \\
\text { prostate cancer }\end{array}$} \\
\hline Yes & 80 & 14.7 & & $6.4-22.9$ & 80 & 18.3 & & $9.1-27.4$ & 263 & 44.4 & & $37.5-51.2$ & 423 & 34.4 & & $29.7-39.0$ \\
\hline No & 1316 & 6.9 & 0.069 & $5.5-8.4$ & 1175 & 10.8 & 0.117 & $8.7-12.8$ & 4294 & 33.4 & 0.002 & $31.8-35.1$ & 6785 & 24.6 & 0.000 & $23.4-25.8$ \\
\hline \multicolumn{17}{|l|}{ Ever had a PSA test } \\
\hline All & 1418 & 16.1 & & $13.8-18.3$ & 1277 & 24.7 & & $21.9-27.5$ & 4661 & 56.8 & & $55.2-58.3$ & 7356 & 43.7 & & $42.4-44.9$ \\
\hline \multicolumn{17}{|l|}{ Race } \\
\hline White & 1133 & 15.6 & & $13.2-18.1$ & 1020 & 24.2 & & $21.0-27.3$ & 3886 & 58.1 & & $56.5-59.8$ & 6039 & 44.4 & & $43.1-45.6$ \\
\hline African-American & 158 & 25.7 & 0.038 & $16.7-34.6$ & 165 & 29.8 & 0.154 & $22.6-37.0$ & 515 & 52.6 & 0.035 & $47.9-57.4$ & 838 & 43.7 & 0.765 & $39.4-48.0$ \\
\hline $\begin{array}{l}\text { American Indian, } \\
\text { Alaskan Native }\end{array}$ & 10 & 0.0 & e & e & 11 & 46.4 & 0.243 & $9.4-83.4$ & 33 & 45.7 & 0.100 & $31.1-60.4$ & 54 & 36.8 & 0.178 & $25.8-47.7$ \\
\hline Asian & 40 & 9.5 & 0.201 & $0.3-18.6$ & 33 & 18.7 & 0.415 & $6.0-31.5$ & 104 & 32.1 & 0.000 & $24.7-39.6$ & 177 & 25.3 & 0.000 & $19.9-30.7$ \\
\hline Other & 77 & 10.2 & 0.173 & $2.8-17.6$ & 48 & 20.9 & 0.636 & $7.9-33.9$ & 123 & 42.6 & 0.002 & $33.1-52.1$ & 248 & 32.4 & 0.001 & $25.6-39.2$ \\
\hline \multicolumn{17}{|l|}{$\begin{array}{l}\text { Family history of } \\
\text { prostate cancer }\end{array}$} \\
\hline Yes & 80 & 32.3 & & $21.3-43.4$ & 81 & 42.9 & & $30.1-55.7$ & 266 & 72.2 & & $66.3-78.1$ & 427 & 59.7 & & $55.2-64.2$ \\
\hline No & 1317 & 15.3 & 0.003 & $13.0-17.5$ & 1177 & 23.3 & 0.003 & $20.4-26.1$ & 4321 & 55.8 & 0.000 & $54.2-57.4$ & 6815 & 42.6 & 0.000 & $41.4-43.9$ \\
\hline \multicolumn{17}{|c|}{ Had any PSA test in the past year } \\
\hline All & 1417 & 9.1 & & $7.5-10.7$ & 1,274 & 13.3 & & $11.1-15.4$ & 4630 & 39.2 & & $37.6-40.8$ & 7,321 & 29.1 & & $28.0-30.3$ \\
\hline \multicolumn{17}{|l|}{ Race } \\
\hline White & 1132 & 8.7 & & $6.9-10.5$ & 1,019 & 12.6 & & $0.2-15.0$ & 3859 & 40.2 & & $28.5-41.9$ & 6,010 & 29.6 & & $28.4-30.8$ \\
\hline African-American & 158 & 14.9 & 0.101 & $7.9-22.0$ & 164 & 18.6 & 0.083 & $2.3-24.9$ & 513 & 34.4 & 0.017 & $29.9-38.9$ & 835 & 28.1 & 0.414 & $24.4-31.7$ \\
\hline $\begin{array}{l}\text { American Indian, } \\
\text { Alaskan Native }\end{array}$ & 10 & 0.0 & e & e & 11 & 35.8 & 0.195 & $1.0-70.6$ & 32 & 27.9 & 0.096 & $13.5-42.4$ & 53 & 23.8 & 0.322 & $12.2-35.3$ \\
\hline Asian & 40 & 5.6 & 0.439 & $0.0-13.1$ & 32 & 11.2 & 0.794 & $1.3-21.2$ & 104 & 24.9 & 0.000 & $18.1-31.8$ & 176 & 18.7 & 0.000 & $13.9-23.6$ \\
\hline Other & 77 & 8.2 & 0.899 & $1.7-14.7$ & 48 & 7.4 & 0.189 & $0.0-14.8$ & 122 & 32.0 & 0.085 & $22.8-41.2$ & 247 & 23.0 & 0.042 & $16.9-29.2$ \\
\hline \multicolumn{17}{|l|}{$\begin{array}{l}\text { Family history of } \\
\text { prostate cancer }\end{array}$} \\
\hline Yes & 80 & 17.3 & & $8.4-26.2$ & 80 & 21.6 & & $1.7-31.5$ & 263 & 53.7 & & $46.7-60.7$ & 423 & 41.4 & & $36.5-46.3$ \\
\hline No & 1316 & 8.7 & 0.065 & $7.1-10.3$ & 1,175 & 12.7 & 0.084 & $0.5-14.9$ & 4,294 & 38.3 & 0.000 & $36.6-40.0$ & 6,785 & 28.4 & 0.000 & $27.1-29.6$ \\
\hline
\end{tabular}




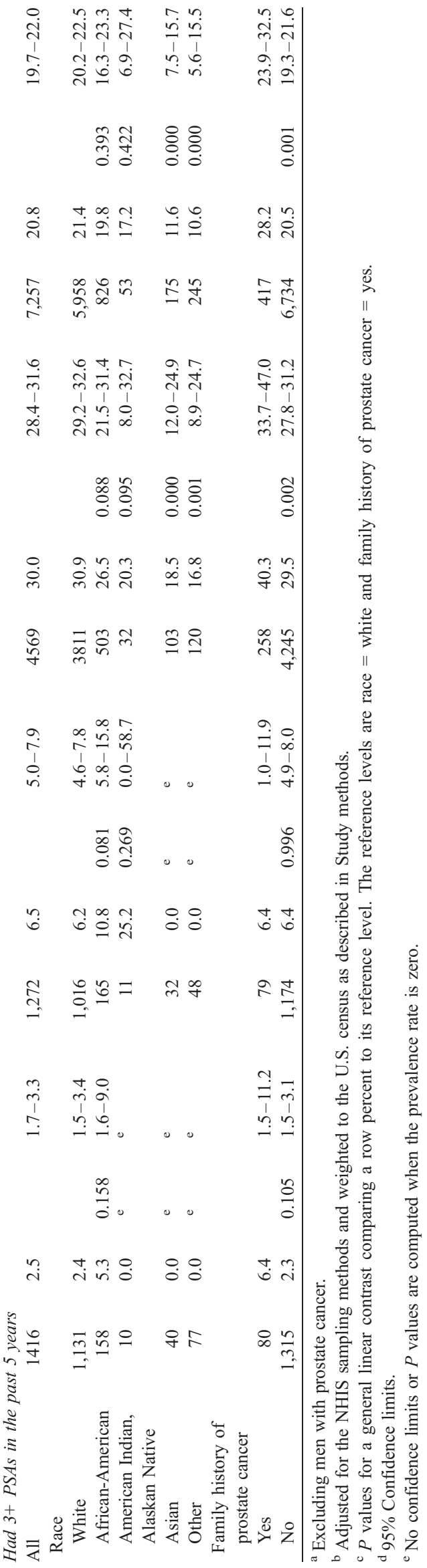

treatment are less likely to extend their life expectancy. However, we found that screening was not inversely related to the number of chronic conditions. As expected, use of PSA testing was greater among men who reported a family history of prostate cancer. Men with a family history have been encouraged to consider testing, and family history has been found related to increased PSA test use in one other study [24].

Our findings on PSA test use by both age and race/ ethnicity are generally consistent with the literature $[13,14,17-19,22,26]$. Overall, use increased until age 80 and then declined, and use was lower among Hispanic men and among Asian/Pacific Islander men. An earlier study found lower PSA test use among African-American men than among white men [18], but more recent studies have not $[10,14,17]$. One report indicates that use in AfricanAmerican men has been increasing more rapidly than in white men [9]. Our findings were mixed, with somewhat greater use by African-American men than others in their $40 \mathrm{~s}$, and similar or lower use by African-American men in their 50s compared with white men. The variation in racial differences in test use by age suggests that important associations might be missed unless racial differences in test use are examined by age.

This study has some attributes that add to its ability to contribute to the existing literature. Data are from a large, nationally representative survey with a good response rate. Experienced, well-trained census interviewers conducted in-home interviews using show cards. Information included PSA test use over a 5-year period as well as recent use and the reason for recent use. Information was also available on several predictive factors that have not been examined in earlier research on PSA testing. Finally, because the survey over-sampled African-American and Hispanic populations, it produced more precise estimates of use.

The study also has several limitations. Most important is the reliance on self-report of PSA test use. Studies that compared self-reported PSA testing with medical records found that patients were aware of only $74-76 \%$ of the tests recorded in medical records, and $27-35 \%$ of men reported having had tests that were not recorded in medical records $[42,43]$. Blood specimens are taken for many different reasons and all of the tests performed on a blood sample may not always be discussed with the patient. In addition, people may underestimate how much time has passed since their last PSA test [44]. The extent of misreporting can also vary by personal and social characteristics and this variation could lead to bias in observed relations between those characteristics and test use $[42,45]$. Although NHIS questions did not allow us to determine the reason for all PSA tests during the previous 5 years, patterns of routine test use were similar to patterns of screening test use. A final limitation of the NHIS survey data is that although it was a large survey and some groups were over-sampled, small sample sizes limited our ability to generate precise estimates 
for some groups, especially American Indian/Alaska Native and Asian populations.

The findings of this study and other PSA test use studies have important implications for medical and public health professionals. First, the methods by which PSA testing has been promoted among men, especially African-American men, may be useful for promoting the use of preventive services that are more clearly beneficial. Historically, access to or use of health services has been lower among men than among women and lower among African-Americans than among white men and women $[26,46,47]$. The level of PSA test use among men, combined with the findings that men are no more likely than women to underuse colorectal cancer screening, suggests that men may be reachable with other preventive services [26]. Similarly, recent research on PSA test use suggests that its use among African-Americans may not be very different from use among white men $[10,17,23,26,47-49]$. The greater use of the PSA test among younger African-American men may signify the success of campaigns to make them aware of their higher risk of prostate cancer at younger ages and to encourage PSA screening [29]. More research is needed to understand strategies that contribute to these successes; the results of such research could be used to improve efforts to promote the use of effective preventive services.

Our findings and those from the literature also raise the question of how decisions are made about the use of PSA as a screening test. The level of PSA screening test use and patterns of use, which are similar to other cancer screening tests, suggests that for some men and their clinicians, PSA may be a routine cancer screening test. This possibility is supported by research showing that many physicians consider PSA screening to be the standard of care [16]. In addition, since about one-fourth of men in their $80 \mathrm{~s}$ and a similar proportion of men apparently in poor health are being screened, some clinicians may be using the test more broadly than is generally recommended.

Although the major medical associations differ in their support of PSA screening recommendations, they all suggest that clinicians should use a process of shared decisionmaking that includes discussing with patients the risks of prostate cancer, the potential benefits and harms of screening, and involving the patients in the decision $[29,50]$. Shared decision-making is recommended because there is no firm evidence or general agreement that the benefits of PSA screening outweigh the harms [1-6,29]. By 20052008, two large-scale randomized screening trials, the NCIsponsored Prostate, Lung, Colorectal and Ovary (PLCO) cancer trial in the United States [51] and the European Randomized Screening for Prostate Cancer (ERSPC) trial in Europe [52], will provide more definitive information on the effects of screening on prostate cancer. These trials are aimed at assessing whether screening reduces deaths from prostate cancer.

The higher level of use of the PSA test for prostate cancer screening may be explained by several factors, including more supportive recommendations by some organizations including the American Cancer Society (ACS) [5]. ACS also recommends a form of shared decision-making for prostate cancer screening. Many clinicians follow ACS recommendations [53-55].

Survey research suggests that most medical practitioners are aware of the screening controversy but think that PSA screening is efficacious $[16,56]$. The extent to which medical providers are aware of the harms and the inconsistencies in the evidence of effectiveness is uncertain [6,57], and recent studies indicate that men too have many misconceptions about prostate cancer screening [58]. Other factors that may contribute to PSA screening include the ease of ordering the blood test, patient interest, reports in medical journals about effectiveness, publicity in the mass media, and celebrity endorsements of screening [16,53,56].

Because of the disagreements about prostate cancer screening, the Centers for Disease Control and Prevention (CDC) organized a conference to recommend public health approaches to prostate cancer [59]. The main recommendation was that agencies provide information needed for informed decision-making to the public and to clinicians [59]. CDC has several related projects underway, and informed decision-making aids are available at CDCs web site www.cdc.gov/cancer/prostate. In addition, major medical associations have this type of information available on their web sites [29], and the U.S. Preventive Services Task Force just released a detailed review and recommendations $[1,2]$.

In conclusion, the levels and patterns of PSA test use suggest that some men and their clinicians may be adopting PSA testing as general practice as they have other screening tests. Like the results of some other recent studies of PSA test use $[6,57,58]$, our findings raise questions about how screening decisions are made. More information is needed on how knowledgeable medical professionals and patients are of the uncertainties about prostate cancer screening and how decisions are being made.

\section{References}

[1] U.S. Preventive Services Task Force. Screening for prostate cancer: recommendation and rationale. Ann Intern Med 2002;137: 915-6.

[2] Harris R, Lohr KN. Screening for prostate cancer: an update of the evidence for the U.S. Preventive Services Task Force. Ann Intern Med 2002;137:917-29.

[3] Etzioni R, Penson DF, Legler JM, Tommaso DD, Boer R, Gann PH, et al. Overdiagnosis due to prostate-specific antigen screening: lessons from U.S. prostate cancer incidence trends. J Natl Cancer Inst 2002;94:981-90.

[4] Thompson I, Carroll P, Coley C, Sweat G, McLeod D, Shellhammer P. Prostate-specific antigen (PSA) best practice policy [commentary]. Oncology 2000;14:267-86.

[5] American Cancer Society. Cancer facts and figures. Atlanta; 2002.

[6] Ransohoff DF, Collins MM, Fowler FJ. Why is prostate cancer screening so common when the evidence is so uncertain? A system without negative feedback. Am J Med 2002;13:663-7. 
[7] Sirovich BE, Schwartz LM, Woloshin S. Screening men for prostate and colorectal cancer in the United States: does practice reflect the evidence? JAMA 2003;289:1414-20.

[8] Potosky AL, Feur EJ, Levin DL. Impact of screening on incidence and mortality of prostate cancer in the United States. Epidemiol Rev 2001;23:181-6.

[9] Etzioni R, Berry KM, Legler JM, Shaw P. Prostate-specific antigen testing in black and white men: an analysis of medicare claims from 1991-1998. Urology 2002;59:251-5.

[10] McDavid K, Melnick TA, Derderian H. Prostate cancer screening trends of New York state men at least 50 years of age, 1994-1997. Prev Med 2000;31:195-202.

[11] Close DR, Kristal AR, Li S, Patterson RE, White E. Associations of demographic and health-related characteristics with prostate cancer screening in Washington state. Cancer Epidemiol Biomarkers Prev 1998;7:627-30.

[12] Centers for Disease Control and Prevention. Screening with the prostate specific antigen test-Texas. Morb Mortal Wkly Rep 1997;49: $818-20$.

[13] Legler JM, Feur EJ, Potosky AL, Merrill RM, Kramer BS. The role of prostate-specific antigen testing patterns in the recent prostate cancer incidence decline in the U.S. Cancer Causes Control 1998;9:519-27.

[14] Collins MM, Stafford RS, Barry MJ. Age specific patterns of prostate specific antigen testing among primary care physicians. J Fam Pract 2000;49:169-72.

[15] Fowler FJ, Bin L, Collins MM, Roberts RG, Oesterling JE, Wasson $\mathrm{JH}$, et al. Prostate cancer screening and beliefs about treatment efficacy: a national survey of primary care physicians and urologists. Am J Med 1998;104:526-32.

[16] Voss ID, Schectman JM. Prostate cancer screening practices and beliefs: a longitudinal physician survey. J Gen Intern Med 2001; 16:831-7.

[17] Steele CB, Miller DS, Maylahn C, Uhler RJ, Baker C. Knowledge, attitudes, and screening practices among older men regarding prostate cancer. Am J Public Health 2000;90:1595-600.

[18] Demark-Wahnefried W, Strigo T, Conaway M, Brunetti M, Rimer BK, Robertson N. Knowledge, beliefs, and prior screening behavior among blacks and whites reporting for prostate cancer screening. Adult Urology 1995;46:346-51.

[19] Brown ML, Potosky AL, Thompson GB, Kessler LG. The knowledge and use of screening tests for colorectal and prostate cancer: data from the 1987 NHIS survey. Prev Med 1990;19:562-74.

[20] Myers RE, Wolf TA, McKee L, McGrory G, Burgh DY, Nelson G. Factors associated with intention to undergo annual prostate cancer screening among African American men in Philadelphia. Cancer 1996;78:471-9.

[21] Eisen SA, Waterman B, Skinner CS, Scherrer JF, Romeis JC, Bucholz K, et al. Sociodemographic and health status characteristics associated with prostate cancer screening. Adult Urology 1999; 53:516-22.

[22] McCoy CB, Anwyl RS, Metsch LR, Inciardi JA, Smith SA, Correa R. Prostate cancer in Florida: knowledge, attitudes, practices, and beliefs. Cancer Pract 1995;3:88-93.

[23] Weinrich SP, Boyd MD, Weinrich M, Greene F, Reynolds WA, Metlin C. Increasing prostate cancer screening in African American men with peer-educator and client-navigator interventions. J Cancer Educ 1998;13:213-9.

[24] Taylor KL, DiPlacido J, Redd WH, Faccenda K, Greer L, Perimutter A. Demographics, family histories, and psychological characteristics of prostate carcinoma screening. Cancer 1998;85:1305-12.

[25] Weller D, Pinnock C, Silagy S, Hiller JE, Marshall VR. Cancer testing in South Australian men: practices and beliefs. Aust N Z J Public Health 1998;22:400-2.

[26] Swan J, Breen N, Coates RJ, Rimer BK, Lee NC. Progress in cancer screening practices in the United States: results from the $2000 \mathrm{Na}-$ tional Health Interview Survey. Cancer 2002;97:1528-40.

[27] Conlisk EA, Lengerich EJ, Demark-Wahnefried W, Schildkraut JM,
Aldrich TE. Prostate cancer: demographic and behavioral correlates of stage at diagnosis among blacks and whites in North Carolina. Adult Urology 1999;53:1194-9.

[28] National Center for Health Statistics. National Health Interview Survey: NHIS Survey Description, Hyattsville, MD; 2001. Available at: http://www.cdc.gov/nchs/nhis.htm\#2000_NHIS.

[29] National Guideline Clearinghouse (NGC). Guideline synthesis: screening for prostate cancer. National Guideline Clearinghouse (NGC) [website]. Rockville (MD): 1998 Dec 28 (updated 2003). [accessed 2003 Apr 16]. Available at: http://www.guideline.gov.

[30] Bloom JR, Grazier K, Hodge F, Hayes WA. Factors affecting the use of screening mammography among African American women. Cancer Epidemiol Biomarkers Prev 1991;1:75-82.

[31] White E, Urban N, Taylor V. Mammography utilization, public health impact, and cost-effectiveness in the United States. Annu Rev Public Health 1993;14:605-33.

[32] Schoen RE, Marcus M, Braham RL. Factors associated with the use of screening mammography in a primary care setting. J Community Health 1994;19:239-52.

[33] U.S. Census Bureau. Current Population Survey: Poverty 2000. Washington (DC): U.S. Department of Commerce; 2001.

[34] Shah BV, Barnwell BG, Bieler GS. SUDAAN. Research Triangle Park (NC): Research Triangle Institute; 1997.

[35] Menard S. Applied logistic regression analysis. Thousand Oaks (CA): Sage; 2001

[36] Graubard BI, Korn EL. Predictive margins with survey data. Biometrics 1999;55:652-9.

[37] Rothman KJ, Greenland S. Modern epidemiology. Second ed. Philadelphia: Lippincott-Raven; 1998.

[38] U.S. Preventive Services Task Force. Guide to clinical preventive services. third edition: periodic updates. Rockville, MD Agency for Healthcare Research and Quality and the U.S. Public Health Service, 2003.

[39] Smith RA, Von Eschenbach AC, Wender R, Levin B, Byers T, Rothenberger D. American Cancer Society guidelines for the early detection of cancer: update of early detection guidelines for prostate, colorectal, and endometrial cancers. CA Cancer J Clin 2001;51: $38-75$.

[40] Winawer SJ, Fletcher RH, Miller L. Colorectal cancer screening: clinical guidelines and rationale. Gastroenterology 1997;112:594-642.

[41] Rakowski W, Rimer BK, Bryant SA. Integrating behavior and intention regarding mammography by respondents in the 1990 National Health Interview Survey of health promotion and disease prevention. Public Health Rep 1993;108:605-24.

[42] Jordan TR, Price JH, King KA, Masyk T, Bedell AW. The validity of male patients' self-reports regarding prostate cancer screening. Prev Med 1999;28:297-303.

[43] Volk RJ, Cass AR. The accuracy of primary care patients' self-reports of prostate-specific antigen testing. Am J Prev Med 2002;22:56-8.

[44] Gordon NP, Hiatt RA, Lampert DI. Concordance of self-reported data and medical record audit for six cancer screening procedures. J Natl Cancer Inst 1993;85:566-70.

[45] Paskett ED, Tatum CM, Mack DW, Hoen H, Case LD, Valez R. Validation of self-reported breast and cervical cancer screening tests among low-income minority women. Cancer Epidemiol Biomarkers Prev 1996;5:721-6.

[46] Weir R, Browne G, Tunks E, Gafni A, Roberts J. Gender differences in psychosocial adjustment to chronic pain and expenditures for health care services used. Clin J Pain 1997;12:277-90.

[47] Breen N, Kessler LG. Trends in cancer screening: United States. Oncology 1996;10:328-30.

[48] Collins MM. Increasing prostate cancer awareness in African American men. Oncol Nurs Forum 1997;24:91-5.

[49] Breen N, Wagener DK, Brown ML, Davis WW, Ballard-Rarbash R. Progress in cancer screening over a decade: results of cancer screening from the 1987, 1992, 1998 National Health Interview Surveys. J Natl Cancer Inst 2001;93:1704-13. 
[50] Woolf SH. Should we screen for prostate cancer? Men over 50 have a right to decide. BMJ 1997;314:989-90.

[51] Prorok PC, Andriole GL, Bresalier RS, Buys SS, Chia D, Crawford ED, et al. Design of the prostate, lung, colorectal and ovarian (PLCO) cancer screening trial. Control Clin Trials 2000;21: 273S-309S.

[52] Standaert B, Denis L. The European randomized study of screening for prostate cancer: an update. Cancer 1997;80:1830-4.

[53] Austin OJ, Valente S, Hasse LA, Kues JR. Determinants of prostatespecific antigen test use in prostate cancer screening by primary care physicians. Arch Fam Med 1997;6:453-8.

[54] Lawson DA, Simoes EJ, Sharp D, Murayi T, Hagan R, Brownson R, et al. Prostate cancer screening-A physician survey in Missouri. J. Commun Health 1998;23:347-58.

[55] Moran WP, Cohen SJ, Preisser JS, Wofford JL, Sheldon BJ,
McClatchey MW. Factors influencing use of the prostate-specific antigen screening test in primary care. Am J Manag Care 2000;6: $315-24$

[56] Hoffman RM, Paperfuss MR, Buller DB, Moon TE. Attitudes and practices of primary care physicians for prostate cancer screening. Am J Prev Med 1996;12:277-81.

[57] Wilt T. Uncertainty in prostate cancer care: the physician's role in clearing the confusion. JAMA 2000;283:3258-60.

[58] Taylor KL, Shelby R, Kerner J, Redd W, Lynch J. Impact of undergoing prostate carcinoma screening on prostate carcinoma-related knowledge and distress. Cancer 2002;95:1037-44.

[59] Centers for Disease Control and Prevention. U.S. Department of Health and Human Services, Prostate Cancer Conference report: future directions for public health practice and research in prostate cancer. Atlanta, 2002. 